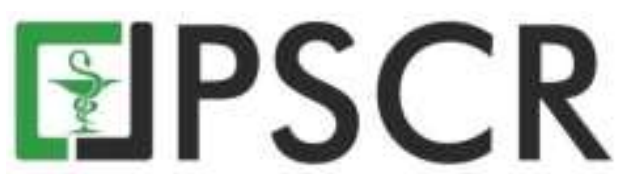

\title{
Postoperative Orthopedic Surgical Site Infection Antibiogram of dr. Soebandi Hospital, Jember in 2019
}

\author{
Nurul Indah Saffanah ${ }^{1}$, Dini Agustina ${ }^{2 *}$ dan Ika Rahmawati Sutejo ${ }^{3}$ \\ ${ }^{1}$ Medicine Faculty, University of Jember, Jl. Kalimantan No. 37, Kampus Tegalboto, Jember, Jawa Timur, \\ Indonesia, 68121 \\ ${ }^{2}$ Microbiology Department, Medicine Faculty, University of Jember, J1. Kalimantan No. 37, Kampus \\ Tegalboto, Jember, Jawa Timur, Indonesia, 68121 \\ ${ }^{3}$ Biochemistry Department, Medicine Faculty, University of Jember, Jl. Kalimantan No. 37, Kampus \\ Tegalboto, Jember, Jawa Timur, Indonesia, 68121 \\ *email korespondensi: dini_agustina@unej.ac.id \\ Received 04 June, 2020, Accepted 12 October 2020, Published 30 October 2020
}

\begin{abstract}
Surgical site infection (SSI) is a microbial infection of the surgical wound within 30 days of operation or one year after surgery if an implant is placed in a patient. The incidence level of postoperative orthopedic SSI range to $71 \%$. An increase in antibiotic-resistant bacteria causes the treatment of postoperative orthopedic surgical wound infections to be less effective, so there is an increase in morbidity, mortality, length of stay, and economic burden on hospital resources. Antibiogram were used to guide the clinician and pharmacist selected the best empiric antimicrobial treatment in the event of pending microbiology culture and susceptibility results to overcome the disadvantages of antibotic-resistant bacteria. This study aimed to form a postoperative orthopedic SSI antibiogram of dr. Soebandi Hospital in Jember City, East Java, Indonesia which can be used as a reference to rational therapy. This study used a descriptive observational study research design with medical records from January - December 2019. A total of 34 isolates were found from 33 patients who did a culture examination. Of the 34 isolates, 25 were found to be positive for bacterial growth, while nine were negative. Twentytwo bacteria were Gram-negative and others were Gram-positive. The bacteria were tested against beta-lactam and non-beta lactam antibiotics. From antibiogram, showed that Gramnegative bacteria sensitive to the meropenem and resistant to cefotaxime. While, gram-positive bacteria showed sensitivity to doxycycline and resistant to erythromycin and penicillin.
\end{abstract}

Keywords: Antibiogram; Surgical Site Infection; Orthopedic Surgery

\section{Introduction}

Surgical site infection (SSI) is a microbial infection of the surgical wound within 30 days of operation or one year after surgery if there is an implant inserted in the patient. Surgical site infections are one of the nosocomial infections that often occur besides pneumonia, urinary tract infections, and bacteremia. Surgical site infection ranks second as the most common infection experienced by surgical patients, and the incidence level of postoperative orthopedic SSI range to $71 \%$. Surgical site infections can cause an increase in 
morbidity, mortality, extended hospital in-patient stays, and economic burden to hospital resources (Amaradeep et al, 2017).

A previous study at King Fahd Hospital of the University, Saudi Arabia, stated that the most common infective organism causing SSI in orthopedic surgery was Staphylococcus spp. including Methicillin-Resistant Staphylococcus aureus (MRSA) (29,11\%), Acinetobacter spp. (21,5\%), Pseudomonas spp. (18\%), and Enterococcus spp. (17,7\%) (AlMulhim et al, 2014). At present, for prophylactic, orthopedic surgery still gives recommendation first-generation and second-generation cephalosporins such as Cefazolin and Cefuroxime. However, if the patient has a history of cephalosporin allergy, Clindamycin, or Vancomycin can be considered. Because of the changes in skin's normal flora and increased prevalence of multidrug-resistant bacteria in hospitals, the treatment becomes ineffective and not be able to provide adequate prevention, so the case of surgical site infection still appear (Li et al, 2013).

To overcome the antibiotic resistance, Indonesia took a role by releasing the Antimicrobial Resistance Control Program based on Regulation of Indonesia Health Minister. The program regulates antibiotic selection based on microbiological examination results, or microbial and antibiotic susceptibility patterns, and directed towards narrowspectrum antibiotics to reduce the development of resistant bacteria. The pattern of bacterial sensitivity to antibiotics can be seen on an antibiogram. The antibiogram consists of the identification of bacteria and testing for sensitivity to antibiotics. With an antibiogram that refers to the results of previous examinations, the physician can provide rational therapy in cases of postoperative orthopedic SSI and resistance to antibiotics can be prevented. Study in the antibiogram of all postoperative orthopedic patients related data in dr. Soebandi Hospital, Jember has not been done yet. This will make the result more reliable and for predicting treatment failure. Also, the presence of antibiogram study in this journal can increase the awareness of the community to not consuming antibiotics without prescription so the antibiotic resistance hopefully didn't happen.

This study aimed to provide information about the sensitivity level of etiological bacteria in surgery site infection of postoperative orthopedic to antibiotics in the form of an antibiogram.

\section{Methods}

This study uses a descriptive retrospective study research design taken in dr. Soebandi Hospital from January - December 2019. The hospital for this study is a regional hospital type $\mathrm{B}$, which mostly the patients domiciled within and around the region. The subject were 
taken by total sampling method. Among 871 orthopedic surgical patients, 33 were included based on inclusion criteria such as experiencing postoperative orthopedic SSI and willing to undergo microbial examination and antibiotic sensitivity tests. Data comprising patientrelated were collected from medical records including age, gender, room while staying in a hospital, the organism causing SSI, and antibiotic susceptibility. Data on the sensitivity of bacterial isolates to antibiotics were obtained from the antibiotic susceptibility test. The result of this test divided into Sensitive (S), Intermediate (I) and Resistant (R). In this study, Percent sensitivity was obtained by dividing the number of isolates that are sensitive to the antibiotic tested $\left(\sum S\right)$ by all strains tested against that antibiotic (n). Similarly, the percentage of resistance was obtained by dividing $\sum R$ by $n$. The more isolates tested (the more $n$ ), the more accurate the percent sensitivity and the percent resistance. Percent sensitivity on the antibiogram is color-coded and numbered if the number is close to 100 or even 100 , the antibiotic is sensitive and recommended for further treatment (Akualing \& Rejeki, 2018).

\section{Results and Discussion}

From 33 patients with postoperative orthopedic SSI, the youngest patient was 14 years old, while the oldest was 63 . The characteristic of the subjects can be seen in Table 1 .

Table 1. Characteristic of subjects as orthopedic surgical patients in dr. Soebandi Hospital from January - December 2019.

\begin{tabular}{lccc}
\hline Variables & Category & Number & Percentage \\
\hline Sex & Male & 25 & $75,7 \%$ \\
& Female & 8 & $24,3 \%$ \\
Age & $12-25$ & 7 & $21,2 \%$ \\
& $26-45$ & 15 & $45,5 \%$ \\
& $46-65$ & 11 & $33,3 \%$ \\
\hline
\end{tabular}

As much as 34 isolates were found on 33 patients, with one patient underwent a double infection. A total of 25 isolates were found positive bacteria, while the rest were negative in bacterial growth. Based on 25 bacteria identified, Gram-negative bacteria were dominated by Escherichia coli, Pantoea spp., Pasteurella pneumotropica, and Pseudomonas aeruginosa. Meanwhile, in Gram-positive bacteria were dominated by Staphylococcaceae families. Table 2 follows the profile of the bacteria identified.

Antibiogram of postoperative orthopedic SSI cases of dr. Soebandi Hospital is classified based on the type of bacteria, namely Gram-negative bacteria tested on 29 antibiotics (Table 3a, 3b and 3c) and Gram-positive bacteria tested on 22 antibiotics (Table $4 \mathrm{a}$ and $4 \mathrm{~b})$. The colors refer to the percent sensitivity of the antibiotic where red indicates low, yellow indicates moderate, and green indicates high sensitivity. 
The level of sensitivity of antibiotics that have been adjusted based on the number of isolates tested (> 50\% isolates) for Gram-negative bacteria from the highest to the lowest were meropenem (93\%), chloramphenicol $(71 \%)$, aztreonam $(11 \%)$, and resistant to cefotaxime $(0 \%)$. Antibiotic sensitivity for gram-positive bacteria from highest to lowest was doxycycline (100\%), trimethoprim-sulfamethoxazole (100\%), and resistant against erythromycin $(0 \%)$ and penicillin $(0 \%)$.

Table 2. Profil of gram-negative and gram-positive bacterial isolated from the antibiotic susceptibility test in dr. Soebandi Hospital from January - December 2019

\begin{tabular}{lllcc}
\hline \multicolumn{1}{c}{ Category } & \multicolumn{1}{c}{ Family } & \multicolumn{1}{c}{ Species } & Frekuensi & \% \\
\hline Gram-negative & Enterobacteriaceae & Escherichia coli & 5 & $20 \%$ \\
22 Isolates & & Enterobacter cloacae & 1 & $4 \%$ \\
$88 \%$ & & Klebsiella oxytoca & 1 & $4 \%$ \\
& & Klebsiella pneumoniae & 1 & $4 \%$ \\
& Erwiniaceae & Pantoea spp. & 2 & $8 \%$ \\
& Moraxellaceae & Acinetobacter & 1 & $4 \%$ \\
& Morganellaceae & Proteus mirabillis & 1 & $4 \%$ \\
& & Providencia rettgeri & 1 & $4 \%$ \\
& Pasteurellaceae & Pasteurella pneumotropica & 3 & $12 \%$ \\
& Pseudomonadaceae & Pseudomonas aeruginosa & 3 & $12 \%$ \\
& & Pseudomonas luteola & 1 & $4 \%$ \\
& Yersiniaceae & Serratia odorifera & 1 & $4 \%$ \\
& & Yersinia pseudotuberculosis & 1 & $4 \%$ \\
\hline Gram-positive & Staphylococcaceae & Staphylococcus aureus & 1 & $4 \%$ \\
Three isolates & & Staphylococcus epidermidis & 1 & $4 \%$ \\
$12 \%$ & & Staphylococcus warneri & 1 & $4 \%$ \\
\hline Total & & & $\mathbf{2 5}$ & $\mathbf{1 0 0 \%}$ \\
\hline
\end{tabular}

The type of bacteria that causes surgical site infections depends on several factors, one of which is the depth of the operation. Superficial surgical wound infections are usually dominated by Gram-positive bacteria, while Gram-negative bacteria are more commonly found in deep surgical wound infection, such as orthopedic surgery (TomaszewskaKowalska et al, 2016).

Escherichia coli (E. coli) became the most prevalent gram-negative bacteria in this study (5 isolates from 22 isolates of Gram-negative bacteria). The presence of gut bacteria in surgical site infection indicates poor hospital hygiene (Negi, 2015). E. coli has the ability to form biofilms and adhere to wounds. In biofilms, bacteria are encased in an extracellular matrix consisting of extracellular polymeric substances, carbohydrate-binding proteins, pili, flagella, and extracellular DNA. This extracellular matrix serves to maintain the survival of bacteria by providing nutrients so that bacteria remain alive. The presence of biofilms in a wound allows the wound harder to heal because biofilms also inhibit host cell immunity. 
The existence of the Quorum sensing mechanism in biofilm makes it possible for bacteria to be resistant to antibiotics. In addition to E. coli, the bacteria Pseudomonas aeruginosa ( $P$. aeruginosa) and Klebsiella pneumoniae (K. pneumoniae) also have the ability to help biofilms (Kostakioti et al, 2013). The formed biofilm allows double infection or mixed infection. In one of the patients in this study, two forming biofilm bacteria were found, namely $K$. oxytoca and Pantoea spp. Quorum sensing in biofilms makes it possible for the two bacteria to exchange information, causing resistance to certain antibiotics (Roy et al, 2014). Pseudomonas aeruginosa was found in this study with three isolates out of 22 Gramnegative bacteria isolates, while $K$. pneumoniae was found in one isolate.

The results of the antibiotic sensitivity test showed that the Enterobacteriaceae bacteria were resistant to beta-lactam antibiotics such as ampicillin, cefazolin, cefuroxime, cefotaxime, and several beta-lactam combination antibiotics. Resistance to beta-lactam groups has been reported where $60 \%$ or more bacteria of $E$. coli and $K$. pneumoniae are resistant to this antibiotic (Iredell et al, 2016). Antibiotic resistance in bacteria can be caused due to virulence factors such as $K$. pneumoniae has capsules, adhesin protein, lipopolysaccharides (LPS), and siderophores in which play a role in the infection process (Agustina et al, 2019). The presence of ESBL and Amp-C as beta-lactamase enzymes can cause antibiotic resistance especially to higher generation cephalosporin class, which in this study were proved resistant to cefotaxime and cefixime (Banerjee et al, 2016). Carbapenem and fluoroquinolon group can be the option if the patient had beta-lactam resistance. Still, cases of resistance to the fluoroquinolone group have been reported, and in this study, the sensitivity of $E$. coli bacteria to levofloxacin and ciprofloxacin was only $40 \%$, while other Enterobacteriaceae 0\%. The incidence of bacteria that become resistant to at least one type of antibiotic in at least three classes of antibiotics is called the Multi-Drug Resistance (MDR) (Estiningsih et al, 2016). The choice of antibiotics to eradicate the MDR bacteria is Carbapenem group.

In other Gram-negative bacteria, found most resistant to aztreonam, ceftazidime, and fluoroquinolone groups such as levofloxacin and ciprofloxacin. The use of trimethoprimsulfamethoxazole proved has sensitivity below 50\%. The presence of MDR bacteria in almost all Gram-negative bacteria in this study shows that the importance of appropriate therapy is done to prevent Extensive Drug Resistance (XDR). XDR occurs when bacteria begin to be resistant to the carbapenem group so it will cause broad antibiotic resistance, not only the beta-lactam group, but other groups such as aminoglycoside, fluoroquinolone, and 
Table 3a. Antibiograms of postoperative orthopedic SSI cases of dr. Soebandi Hospital for Gram-negative Bacteria tested on 10 antibiotics. ATM= Aztreonam, $\mathrm{AMP}=$ Ampicillin, $\mathrm{P}=$ Penicillin, $\mathrm{TIC}=$ Ticarcillin, $\mathrm{KZ}=$ Cefazolin, $\mathrm{CN}=$ Cephalexin, $\mathrm{CFR}=$ Cefadroxil, $\mathrm{CXM}=$ Cefuroxime, $\mathrm{FOX}=$ Cefoxitin,, $\mathrm{CFM}=$ Cefixime, $\mathrm{N}=$ total isolates, $\mathrm{n}=$ tested isolates, $\% \mathrm{~S}=$ antibiotic sensitivity percentage.Colour indicator: <40 ( $\square$ ), 40-60 ( $\square), 61-80(\square), 81-99(\square)$ and $100(\square)$.

\begin{tabular}{|c|c|c|c|c|c|c|c|c|c|c|c|c|c|c|c|c|c|c|c|c|c|}
\hline \multirow{2}{*}{ Mikroorganisme } & \multirow{2}{*}{$\mathbf{N}$} & \multicolumn{2}{|c|}{ ATM } & \multicolumn{2}{|c|}{ AMP } & \multicolumn{2}{|c|}{$\mathbf{P}$} & \multicolumn{2}{|c|}{ TIC } & \multicolumn{2}{|c|}{ KZ } & \multicolumn{2}{|c|}{$\mathbf{C N}$} & \multicolumn{2}{|c|}{ CFR } & \multicolumn{2}{|c|}{ CXM } & \multicolumn{2}{|c|}{ FOX } & \multicolumn{2}{|c|}{ CFM } \\
\hline & & $\mathbf{N}$ & $\% \mathrm{~S}$ & $\mathbf{n}$ & $\% \mathrm{~S}$ & $\mathbf{N}$ & $\% \mathrm{~S}$ & $\mathrm{n}$ & $\% \mathrm{~S}$ & $\mathbf{N}$ & $\% \mathrm{~S}$ & $\mathbf{n}$ & $\% \mathrm{~S}$ & $\mathbf{N}$ & $\% \mathrm{~S}$ & $\mathbf{N}$ & $\% \mathrm{~S}$ & $\mathrm{n}$ & $\% \mathrm{~S}$ & $\mathbf{N}$ & $\% \mathrm{~S}$ \\
\hline E. coli & 5 & 3 & $33,3 \%$ & 5 & $20 \%$ & - & - & - & - & 3 & $0 \%$ & 5 & $80 \%$ & 2 & $0 \%$ & 5 & $0 \%$ & 2 & $0 \%$ & 4 & $0 \%$ \\
\hline P. pneumotropica & 3 & 3 & $0 \%$ & 2 & $50 \%$ & 1 & $0 \%$ & - & - & 1 & $100 \%$ & 2 & $50 \%$ & - & - & 1 & $100 \%$ & 1 & $100 \%$ & 2 & $0 \%$ \\
\hline P. aeruginosa & 3 & 3 & $33,3 \%$ & - & - & - & - & 3 & $0 \%$ & - & - & 3 & $66,6 \%$ & - & - & - & - & - & - & - & - \\
\hline Pantoea spp & 2 & 2 & $0 \%$ & 2 & $0 \%$ & - & - & - & - & 1 & $0 \%$ & 2 & $0 \%$ & - & - & 2 & $0 \%$ & 2 & $50 \%$ & 1 & $0 \%$ \\
\hline Acinetobacter & 1 & - & - & - & - & - & - & - & - & - & - & 1 & $0 \%$ & - & - & - & - & - & - & - & - \\
\hline C. luteola & 1 & 1 & $0 \%$ & - & - & - & - & 1 & $0 \%$ & - & - & 1 & $100 \%$ & - & - & - & - & - & - & 1 & $0 \%$ \\
\hline E. cloacae & 1 & 1 & $0 \%$ & 1 & $0 \%$ & - & - & - & - & 1 & $0 \%$ & 1 & $0 \%$ & - & - & 1 & $0 \%$ & 1 & $0 \%$ & 1 & $0 \%$ \\
\hline K. oxytoca & 1 & 1 & $0 \%$ & 1 & $0 \%$ & - & - & - & - & 1 & $0 \%$ & 1 & $100 \%$ & - & - & 1 & $0 \%$ & 1 & $100 \%$ & 1 & $0 \%$ \\
\hline K. pneumoniae & 1 & 1 & $0 \%$ & 1 & $0 \%$ & - & - & - & - & 1 & $0 \%$ & 1 & $0 \%$ & - & - & 1 & $0 \%$ & 1 & $100 \%$ & - & - \\
\hline P. mirabillis & 1 & - & - & 1 & $0 \%$ & - & - & - & - & - & - & 1 & $100 \%$ & 1 & $0 \%$ & 1 & $0 \%$ & - & - & 1 & $0 \%$ \\
\hline P. rettgeri & 1 & 1 & $0 \%$ & 1 & $0 \%$ & - & - & - & - & 1 & $0 \%$ & 1 & $100 \%$ & - & - & 1 & $100 \%$ & 1 & $100 \%$ & 1 & $100 \%$ \\
\hline S. odorifera & 1 & 1 & $0 \%$ & 1 & $0 \%$ & - & - & - & - & 1 & $0 \%$ & 1 & $0 \%$ & - & - & 1 & $0 \%$ & 1 & $0 \%$ & - & - \\
\hline $\begin{array}{l}Y . \\
\text { pseudotuberculosis }\end{array}$ & 1 & 1 & $0 \%$ & 1 & $100 \%$ & - & - & - & - & 1 & $100 \%$ & 1 & $100 \%$ & - & - & 1 & $100 \%$ & 1 & $100 \%$ & 1 & $0 \%$ \\
\hline
\end{tabular}

Table 3b. Antibiograms of postoperative orthopedic SSI cases of dr. Soebandi Hospital for Gram-negative Bacteria tested on 9 antibiotics. CTX= Cefotaxime, $\mathrm{CAZ}=$ Ceftazidime, $\mathrm{CRO}=$ Ceftriaxone, $\mathrm{IMP}=$ Imipenem, $\mathrm{MEM}=$ Meropenem, $\mathrm{AMC}=$ Amoxicillin-clavulanate, $\mathrm{SAM}=$ Ampicillin-sulbactam, $\mathrm{SXT}=$ Trimethoprim-sulfamethoxazole, $\mathrm{TZP}=$ Piperacillin-tozobactam, $\mathrm{N}=$ total isolates, $\mathrm{n}=$ tested isolates, $\% \mathrm{~S}=$

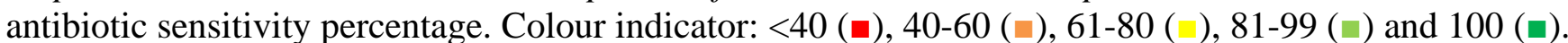

\begin{tabular}{|c|c|c|c|c|c|c|c|c|c|c|c|c|c|c|c|c|c|c|c|}
\hline \multirow{2}{*}{ Mikroorganisme } & \multirow{2}{*}{$\mathbf{N}$} & \multicolumn{2}{|c|}{ CTX } & \multicolumn{2}{|c|}{ CAZ } & \multicolumn{2}{|c|}{ CRO } & \multicolumn{2}{|c|}{ IPM } & \multicolumn{2}{|c|}{ MEM } & \multicolumn{2}{|c|}{ AMC } & \multicolumn{2}{|c|}{ SAM } & \multicolumn{2}{|c|}{ SXT } & \multicolumn{2}{|c|}{ TZP } \\
\hline & & $\mathbf{n}$ & $\% \mathrm{~S}$ & $\mathbf{n}$ & $\% \mathrm{~S}$ & $\mathbf{N}$ & $\% \mathrm{~S}$ & $\mathbf{n}$ & $\% \mathrm{~S}$ & $\mathbf{N}$ & $\% \mathrm{~S}$ & $\mathbf{N}$ & $\% \mathrm{~S}$ & $\mathbf{n}$ & $\% \mathrm{~S}$ & $\mathbf{n}$ & $\% \mathrm{~S}$ & $\mathbf{n}$ & $\% \mathrm{~S}$ \\
\hline E. coli & 5 & 5 & $0 \%$ & 5 & $60 \%$ & 2 & $50 \%$ & 1 & $0 \%$ & 4 & $100 \%$ & 5 & $40 \%$ & 5 & $0 \%$ & 5 & $80 \%$ & 5 & $80 \%$ \\
\hline P. pneumotropica & 3 & 3 & $0 \%$ & 3 & $0 \%$ & 2 & $0 \%$ & - & - & 2 & $100 \%$ & 1 & $100 \%$ & 1 & $100 \%$ & 3 & $66,6 \%$ & 3 & $33,3 \%$ \\
\hline P. aeruginosa & 3 & - & - & 3 & $100 \%$ & - & - & - & - & - & - & - & - & - & - & - & - & 3 & $100 \%$ \\
\hline Pantoea spp & 2 & 2 & $0 \%$ & 2 & $0 \%$ & 2 & $0 \%$ & - & - & 2 & $50 \%$ & 2 & $50 \%$ & 2 & $0 \%$ & 2 & $50 \%$ & 2 & $50 \%$ \\
\hline Acinetobacter & 1 & 1 & $0 \%$ & 1 & $0 \%$ & - & - & - & - & - & - & - & - & 1 & $0 \%$ & 1 & $100 \%$ & 1 & $0 \%$ \\
\hline C. luteola & 1 & - & - & 1 & $100 \%$ & - & - & - & - & - & - & - & - & - & - & - & - & 1 & $100 \%$ \\
\hline E. cloacae & 1 & 1 & $0 \%$ & 1 & $0 \%$ & 1 & $0 \%$ & - & - & 1 & $100 \%$ & 1 & $0 \%$ & - & - & 1 & $0 \%$ & 1 & $0 \%$ \\
\hline К. oxytoca & 1 & - & - & 1 & $0 \%$ & 1 & $0 \%$ & - & - & 1 & $100 \%$ & 1 & $0 \%$ & 1 & $0 \%$ & 1 & $0 \%$ & 1 & $100 \%$ \\
\hline K. pneumoniae & 1 & 1 & $0 \%$ & 1 & $0 \%$ & - & - & - & - & - & - & 1 & $0 \%$ & 1 & $0 \%$ & 1 & $0 \%$ & 1 & $100 \%$ \\
\hline
\end{tabular}


Table 3b. Antibiograms of postoperative orthopedic SSI cases of dr. Soebandi Hospital for Gram-negative Bacteria tested on 9 antibiotics. CTX= Cefotaxime, $\mathrm{CAZ}=$ Ceftazidime, $\mathrm{CRO}=$ Ceftriaxone, $\mathrm{IMP}=$ Imipenem, $\mathrm{MEM}=$ Meropenem, $\mathrm{AMC}=$ Amoxicillin-clavulanate, $\mathrm{SAM}=$ Ampicillin-sulbactam, $\mathrm{SXT}=$ Trimethoprim-sulfamethoxazole, $\mathrm{TZP}=$ Piperacillin-tozobactam, $\mathrm{N}=$ total isolates, $\mathrm{n}=$ tested isolates, $\% \mathrm{~S}=$

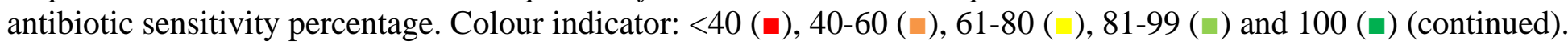

\begin{tabular}{|c|c|c|c|c|c|c|c|c|c|c|c|c|c|c|c|c|c|c|c|}
\hline \multirow{2}{*}{ Mikroorganisme } & \multirow{2}{*}{$\mathbf{N}$} & \multicolumn{2}{|c|}{ CTX } & \multicolumn{2}{|c|}{ CAZ } & \multicolumn{2}{|c|}{ CRO } & \multicolumn{2}{|c|}{ IPM } & \multicolumn{2}{|c|}{ MEM } & \multicolumn{2}{|c|}{ AMC } & \multicolumn{2}{|c|}{ SAM } & \multicolumn{2}{|c|}{ SXT } & \multicolumn{2}{|c|}{ TZP } \\
\hline & & $\mathbf{n}$ & $\% \mathrm{~S}$ & $\mathrm{n}$ & $\% \mathrm{~S}$ & $\mathbf{N}$ & $\% \mathrm{~S}$ & $\mathbf{n}$ & $\% \mathrm{~S}$ & $\mathbf{N}$ & $\% \mathrm{~S}$ & $\mathbf{N}$ & $\% \mathrm{~S}$ & $\mathbf{n}$ & $\% \mathrm{~S}$ & $\mathbf{n}$ & $\% \mathrm{~S}$ & $\mathbf{n}$ & $\% \mathrm{~S}$ \\
\hline Proteus mirabillis & 1 & 1 & $0 \%$ & 1 & $100 \%$ & - & - & - & - & 1 & $100 \%$ & 1 & $0 \%$ & 1 & $0 \%$ & 1 & $0 \%$ & 1 & $100 \%$ \\
\hline $\begin{array}{l}\text { Providencia } \\
\text { rettgeri }\end{array}$ & 1 & 1 & $0 \%$ & 1 & $0 \%$ & 1 & $0 \%$ & - & - & 1 & $100 \%$ & 1 & $0 \%$ & 1 & $0 \%$ & 1 & $0 \%$ & 1 & $0 \%$ \\
\hline S. odorifera & 1 & 1 & $0 \%$ & 1 & $0 \%$ & - & - & - & - & - & - & 1 & $0 \%$ & 1 & $0 \%$ & 1 & $0 \%$ & 1 & $0 \%$ \\
\hline $\begin{array}{l}Y . \\
\text { pseudotuberculosis }\end{array}$ & 1 & 1 & $0 \%$ & 1 & $0 \%$ & 1 & $100 \%$ & - & - & 1 & $100 \%$ & 1 & $100 \%$ & 1 & $100 \%$ & 1 & $100 \%$ & 1 & $100 \%$ \\
\hline
\end{tabular}

Table 3c. Antibiograms of postoperative orthopedic SSI cases of dr. Soebandi Hospital for Gram-negative Bacteria tested on 10 antibiotics. TOB= Tobramycin, $\mathrm{K}=$ Kanamycin, $\mathrm{AK}=$ Amikacin, $\mathrm{TE}=$ Tetracycline, $\mathrm{DO}=$ Doxycyclin, $\mathrm{C}=$ Chloramphenicol, $\mathrm{LEV}=$ Levofloxacin, $\mathrm{CIP}=$ Ciprofloxacin, $\mathrm{OFX}=$ Ofloxacin, $\mathrm{CT}=$ Colistin, $\mathrm{N}=$ total isolates, $\mathrm{n}=$ tested isolates, $\% \mathrm{~S}=$ antibiotic sensitivity percentage. Colour

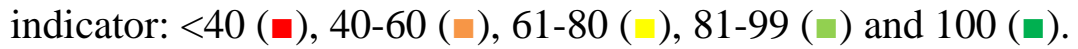

\begin{tabular}{|c|c|c|c|c|c|c|c|c|c|c|c|c|c|c|c|c|c|c|c|c|c|}
\hline \multirow{2}{*}{ Mikroorganisme } & \multirow{2}{*}{$\mathbf{N}$} & \multicolumn{2}{|c|}{ TOB } & \multicolumn{2}{|c|}{$\mathbf{K}$} & \multicolumn{2}{|c|}{$\mathbf{A K}$} & \multicolumn{2}{|c|}{ TE } & \multicolumn{2}{|c|}{ DO } & \multicolumn{2}{|r|}{$\mathbf{C}$} & \multicolumn{2}{|c|}{ LEV } & \multicolumn{2}{|c|}{ CIP } & \multicolumn{2}{|c|}{ OFX } & \multicolumn{2}{|c|}{ CT } \\
\hline & & $\mathbf{n}$ & $\% \mathrm{~S}$ & $\mathbf{n}$ & $\% \mathrm{~S}$ & $\mathbf{n}$ & $\% \mathrm{~S}$ & $\mathbf{n}$ & $\% \mathrm{~S}$ & $\mathbf{N}$ & $\% \mathrm{~S}$ & $\mathbf{n}$ & $\% \mathrm{~S}$ & $\mathbf{n}$ & $\% \mathrm{~S}$ & $\mathbf{N}$ & $\% \mathrm{~S}$ & $\mathbf{N}$ & $\% \mathrm{~S}$ & $\mathbf{n}$ & $\% \mathrm{~S}$ \\
\hline E. coli & 5 & 3 & $66,6 \%$ & 2 & $0 \%$ & 2 & $0 \%$ & 5 & $40 \%$ & 2 & $50 \%$ & 5 & $100 \%$ & 5 & $40 \%$ & 5 & $40 \%$ & - & - & - & - \\
\hline P. pneumotropica & 3 & 3 & $66,6 \%$ & 1 & $0 \%$ & 1 & $0 \%$ & 2 & $0 \%$ & - & - & 3 & $66,6 \%$ & 3 & $33,3 \%$ & 3 & $33,3 \%$ & 1 & $0 \%$ & - & - \\
\hline P. aeruginosa & 3 & 3 & $66,6 \%$ & & - & - & - & - & - & - & - & - & - & 2 & $100 \%$ & 3 & $66,6 \%$ & - & - & 3 & $100 \%$ \\
\hline Pantoea spp & 2 & 2 & $0 \%$ & 1 & $0 \%$ & 1 & $0 \%$ & 2 & $0 \%$ & - & - & 2 & $100 \%$ & 2 & $0 \%$ & 2 & $0 \%$ & - & - & - & - \\
\hline Acinetobacter & 1 & 1 & $0 \%$ & - & - & - & - & 1 & $0 \%$ & 1 & $0 \%$ & - & - & 1 & $0 \%$ & 1 & $0 \%$ & - & - & - & - \\
\hline C. luteola & 1 & 1 & $100 \%$ & - & - & - & - & - & - & - & - & - & - & 1 & $0 \%$ & 1 & $0 \%$ & - & - & 1 & $0 \%$ \\
\hline E. cloacae & 1 & 1 & $0 \%$ & 1 & $100 \%$ & 1 & $100 \%$ & 1 & $0 \%$ & - & - & 1 & $0 \%$ & 1 & $0 \%$ & 1 & $0 \%$ & - & - & - & - \\
\hline K. oxytoca & 1 & 1 & $100 \%$ & 1 & $0 \%$ & 1 & $0 \%$ & 1 & $0 \%$ & - & - & 1 & $100 \%$ & 1 & $0 \%$ & 1 & $0 \%$ & - & - & - & - \\
\hline K. pneumoniae & 1 & 1 & $100 \%$ & 1 & $0 \%$ & 1 & $0 \%$ & 1 & $0 \%$ & - & - & 1 & $0 \%$ & 1 & $0 \%$ & 1 & $0 \%$ & - & - & - & - \\
\hline P. mirabillis & 1 & - & - & - & - & - & - & 1 & $0 \%$ & 1 & $0 \%$ & 1 & $0 \%$ & 1 & $100 \%$ & 1 & $100 \%$ & - & - & - & - \\
\hline P. rettgeri & 1 & 1 & $100 \%$ & 1 & $0 \%$ & 1 & $0 \%$ & 1 & $0 \%$ & - & - & 1 & $0 \%$ & 1 & $0 \%$ & 1 & $0 \%$ & - & - & - & - \\
\hline S. odorifera & 1 & 1 & $0 \%$ & 1 & $0 \%$ & 1 & $0 \%$ & 1 & $0 \%$ & - & - & 1 & $100 \%$ & 1 & $0 \%$ & 1 & $0 \%$ & - & - & - & - \\
\hline $\begin{array}{l}Y . \\
\text { pseudotuberculosis }\end{array}$ & 1 & 1 & $100 \%$ & 1 & $0 \%$ & 1 & $0 \%$ & 1 & $0 \%$ & - & - & 1 & $100 \%$ & 1 & $0 \%$ & 1 & $0 \%$ & - & - & - & - \\
\hline
\end{tabular}


Table 4a. Antibiograms of postoperative orthopedic SSI cases of dr. Soebandi Hospital for Gram-positive Bacteria tested on 10 antibiotics. AMP= Ampicillin, $\mathrm{P}=$ Penicilin, $\mathrm{OX}=$ Oxacillin, $\mathrm{CN}=$ Cephalexin, $\mathrm{CFR}=$ Cefadroxil, $\mathrm{FOX}=$ Cefoxitin, $\mathrm{CFM}=$ Cefixime, $\mathrm{MEM}=$ Meropenem, $\mathrm{AMC}=$ Amoxicillin-clavulanate, $\mathrm{SXT}=$ Trimethoprim-sulfamethoxazole, $\mathrm{N}=$ total isolates, $\% \mathrm{~S}=$ antibiotic sensitivity percentage. $\mathrm{Colour}$ indicator: <40 ( $\square)$, 40-60 ( $\square)$, 61-80 ( $), 81-99(\square)$ and $100(\square)$.

\begin{tabular}{|c|c|c|c|c|c|c|c|c|c|c|c|}
\hline Mikroorganisme & $\mathrm{N}$ & $\begin{array}{c}\text { AMP } \\
\% \mathrm{~S}\end{array}$ & $\begin{array}{c}\mathrm{P} \\
\% \mathrm{~S}\end{array}$ & $\begin{array}{l}\mathrm{OX} \\
\% \mathrm{~S} \\
\end{array}$ & $\begin{array}{l}\mathrm{CN} \\
\% \mathrm{~S}\end{array}$ & $\begin{array}{c}\text { CFR } \\
\% \mathrm{~S} \\
\end{array}$ & $\begin{array}{c}\text { FOX } \\
\% \mathrm{~S}\end{array}$ & $\begin{array}{c}\text { CFM } \\
\% \mathrm{~S} \\
\end{array}$ & $\begin{array}{c}\text { MEM } \\
\% \mathrm{~S}\end{array}$ & $\begin{array}{c}\text { AMC } \\
\% \mathrm{~S}\end{array}$ & $\begin{array}{c}\text { SXT } \\
\% \mathrm{~S} \\
\end{array}$ \\
\hline S. epidermidis & 1 & - & $0 \%$ & - & $0 \%$ & - & $0 \%$ & - & - & - & - \\
\hline S. aureus & 1 & - & $0 \%$ & - & $100 \%$ & - & $0 \%$ & - & - & - & $100 \%$ \\
\hline S. warneri & 1 & $100 \%$ & - & $100 \%$ & - & $100 \%$ & $100 \%$ & $0 \%$ & $100 \%$ & $100 \%$ & $100 \%$ \\
\hline
\end{tabular}

Table 4b. Antibiograms of postoperative orthopedic SSI cases of dr. Soebandi Hospital for Gram-positive Bacteria tested on 12 antibiotics. TOB= Tobramycin, $\mathrm{TE}=$ Tetracycline, $\mathrm{DO}=$ Doxycycline, $\mathrm{DA}=$ Clindamycin, $\mathrm{AZM}=$ Azithromycin, $\mathrm{E}=$ Erythromycin, $\mathrm{LZD}=$ Linezolid, $\mathrm{C}=$ Chloramphenicol, $\mathrm{LEV}=$ Levofloxacyn, $\mathrm{CIP}=$ Ciprofloxacin, $\mathrm{OFX}=$ Ofloxacin, $\mathrm{VA}=$ Vancomycin, $\mathrm{N}=$ total isolates, $\% \mathrm{~S}=$ antibiotic

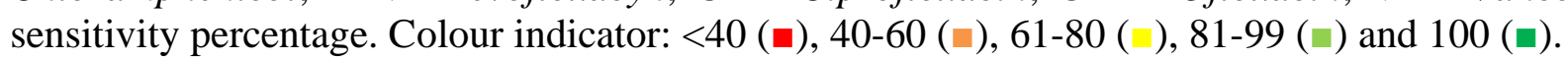

\begin{tabular}{|c|c|c|c|c|c|c|c|c|c|c|c|c|c|}
\hline Mikroorganisme & $\mathrm{N}$ & TOB & $\begin{array}{l}\text { TE } \\
\% \mathrm{~S}\end{array}$ & DO & $\begin{array}{l}\mathrm{DA} \\
\% \mathrm{~S}\end{array}$ & AZM & $\begin{array}{c}\mathrm{E} \\
\% \mathrm{~S}\end{array}$ & LZD & $\begin{array}{c}\mathrm{C} \\
\% \mathrm{~S}\end{array}$ & LEV & $\begin{array}{l}\text { CIP } \\
\% \text { S }\end{array}$ & OFX & $\begin{array}{l}\text { VA } \\
\% S\end{array}$ \\
\hline S. epidermidis & 1 & $0 \%$ & $0 \%$ & $100 \%$ & $0 \%$ & $0 \%$ & $0 \%$ & $0 \%$ & $0 \%$ & $0 \%$ & $0 \%$ & $0 \%$ & $0 \%$ \\
\hline S. aureus & 1 & - & $100 \%$ & $100 \%$ & $0 \%$ & $100 \%$ & $0 \%$ & $100 \%$ & $100 \%$ & $100 \%$ & $100 \%$ & $100 \%$ & $0 \%$ \\
\hline S. warneri & 1 & . & $100 \%$ & $100 \%$ & $100 \%$ & $100 \%$ & - & $100 \%$ & $0 \%$ & $100 \%$ & $100 \%$ & - & $100 \%$ \\
\hline
\end{tabular}


beta-lactamase inhibitors (Banerjee et al, 2016). In this study, 14 bacteria tested for meropenem showed 93\% sensitive results; in which the bacteria caused postoperative orthopedic wound infection in dr. Soebandi Hospital couldn't be categorized as XDR bacteria. Drugs that can be used in MDR bacteria was chloramphenicol, which obtained a sensitivity level of $>70 \%$ tested against 17 isolates.

Gram-positive bacteria found in this study can cause surgical site infections through the mechanism of biofilm formation. S. aureus bacteria are the bacteria that most often causes surgical wound infections to date (Pal et al, 2019). S. epidermidis bacteria in this study indicates that this bacteria has become a pathogen. Although this bacteria is less virulent than S. aureus, it is a pathogen in infections related to implant procedures. In recent years, S. epidermidis has become a major bacterial cause of surgical wound infections in orthopedic implant surgery cases (Takizawa et al, 2017). The presence of $S$. warneri can cause superficial infections or deep infections, such as osteomyelitis. Besides, S. warneri also often found in cases of postoperative surgical wound infection in orthopedic implants due to the presence of locus minoris resistentiae or a condition in the tissues or organs of the body, which decreases its strength making it more susceptible to disease. In implant surgery, the surrounding tissuewill be in direct contact with the new biomaterial. This is one of the factors developing $S$. warneri bacteria (Campoccia et al, 2010).

The Gram-positive bacteria in this study were proven to be MDR bacteria. Effective treatments that have been carried out to deal with these bacteria are doxycycline, which reach $100 \%$ sensitivity of the three isolates tested and combination of trimethoprim-sulfamethoxazole by $100 \%$. Increasing the effectivity of antibiotic against Gram positive bacteria can be through the use of combination antibiotics. Combination of trimethoprim-sulfamethoxazole has a mechanism of action to strengthen each other (synergistically) by inhibiting bacterial folic acid synthesis in which folic acid is needed by bacteria for its survival (Syahputra et al, 2018).

There limitation in this study is the limited number of subjects cannot describe the entire population. The population just based on one hospital, therefore the result can't be generalized in all orthopedic cases in Jember City. But, the existence of antibiogram in one hospital can help the hospital have the right regulation of antibiotic use which can reduce the patient and hospital disadvantages.

\section{Conclusion}

Antibiotics with the highest level of sensitivity to Gram-negative bacteria was meropenem. Meanwhile, the highest level of sensitivity to Gram-positive was Doxyxyclin.

\section{Acknowledgement}


The authors would like to thank the teams of dr. Soebandi Hospital for excellent technical assistance.

\section{Conflict of Interest}

The authors don't have any conflict of interest.

\section{References}

Akualing, J.S. and Rejeki, I.P.S. (2018). Antibiogram. Indonesian Journal Of Clinical Pathology And Medical Laboratory, 23(1): pp.90.

Agustina, D. Nadyatara, K.,Mufida, D.C.,Elfiah, U.,Shodikin, M.A.,and Suswati, E. (2019). Faktor Virulensi Outer Membrane Protein $20 \mathrm{kDa}$ Klebsiella pneumoniae sebagai Protein Hemaglutinin dan Adhesin. eJournal Kedokteran Indonesia (eJKI), 7(3): pp.200-204.

Al-Mulhim, F.A.,Baragbah, M.A.,Sadat-Ali, M.,Alomran, A.S.,and Azam, M.Q. (2014). Prevalence of Surgical Site Infection in Orthopedic Surgery: A 5-year Analysis. International Surgery, 99(3): pp.264-268.

Amaradeep,Shiva Prakah,and Manjappa (2017). Surgical site infections in orthopedic implant surgery and its risk factors: A prospective study in teaching hospital. International Journal of Orthopaedics Sciences, 3(3c): pp.169-172.

Banerjee, A.,Ghosh, M.,Karak, K.,Basu, S.,Mukhopadhyay, B.B.,Mallik, S., and Saha, B. (2016). Lower respiratory tract infection in two tertiary hospitals of Kolkata and carbapenem resistance. International Journal of Infectious Diseases, 45: pp.114.

Campoccia, D.,Montanaro, L.,Visai, L.,Corazzari, T.,Poggio, C.,Pegreffi, F.,Maso, A.,Pirini, V.,Ravaioli, S.,Cangini, I.,Speziale, P.,and Arciola, C.R. (2010). Characterization of 26 Staphylococcus warneri isolates from orthopedic infections. The International Journal of Artificial Organs, 33(9): pp.575-581.

Estiningsih, D.,Puspitasari, I.,and Nuryastuti, T. (2016). Identifikasi Infeksi MultidrugResistant Organisms (MDRO) Pada Pasien Yang Dirawat Di Bangsal Neonatal Intensive Care Unit (Nicu) Rumah Sakit. , 6: pp.6.

Iredell, J.,Brown, J.,and Tagg, K. (2016). Antibiotic resistance in Enterobacteriaceae: mechanisms and clinical implications. BMJ: pp.6420.

Kostakioti, M.,Hadjifrangiskou, M.,and Hultgren, S.J. (2013). Bacterial Biofilms: Development, Dispersal, and Therapeutic Strategies in the Dawn of the Postantibiotic Era. Cold Spring Harbor Perspectives in Medicine, 3(4): pp.010306-010306.

Li, G.,Guo, F.,Ou, Y.,Dong, G.,and Zhou, W. (2013). Epidemiology and outcomes of surgical site infections following orthopedic surgery. American Journal of Infection Control, 41(12): pp.1268-1271.

Negi, V. (2015). Bacteriological Profile of Surgical Site Infections and Their Antibiogram: A Study From Resource Constrained Rural Setting of Uttarakhand State, India. Journal of Clinical And Diagnostic Research.

Pal, S.,Sayana, A.,Joshi, A.,and Juyal, D. (2019). Staphylococcus aureus: A predominant cause of surgical site infections in a rural healthcare setup of Uttarakhand. Journal of Family Medicine and Primary Care, 8(11): pp.3600.

Roy, S.,Elgharably, H.,Sinha, M.,Ganesh, K.,Chaney, S.,Mann, E.,Miller, C.,Khanna, S.,Bergdall, V.K.,Powell, H.M.,Cook, C.H.,Gordillo, G.M.,Wozniak, D.J.,and Sen, C.K. (2014). Mixed-species biofilm compromises wound healing by disrupting epidermal barrier function: Preclinical burn wound biofilm infection. The Journal of Pathology, 233(4): pp.331-343.

Syahputra, R.R.,Agustina, D.,and Wahyudi, S.S. (2018). Pola Kepekaan Bakteri terhadap Antibiotik pada Pasien Infeksi Saluran Kemih di RSD DR. Soebandi Jember. Journal of Agromedicine and Medical Sciences, 4(3): pp.171-177. 
Takizawa, T.,Tsutsumimoto, T.,Yui, M.,and Misawa, H. (2017). Surgical Site Infections Caused by Methicillin-resistant Staphylococcus epidermidis After Spinal Instrumentation Surgery: SPINE, 42(7): pp.525-530.

Tomaszewska-Kowalska, M.,Kołomecki, K.,and Wieloch-Torzecka, M. (2016). Prevalence and Characteristics of Surgical Site Infections Caused by Gram-negative Rod-shaped Bacteria from the Family Enterobacteriacae and Gram-positive Cocci from the Genus Staphylococcus in Patients who Underwent Surgical Procedures on Selected Surgical Wards. Polish Journal of Surgery, 88(5). 10.15.

\section{(c) (1) (2)}

2020 by the authors. Submitted for possible open access publication under the terms and conditions of the Creative Commons Attribution-ShareAlike 4.0 International (CC BY-SA 4.0) license (https://creativecommons.org/licenses/by-sa/4.0/). 